\title{
VECTOR BUNDLES ON FANO 3-FOLDS WITHOUT INTERMEDIATE COHOMOLOGY
}

\author{
Enrique $A R R O N D O$ * \\ $A N D$ \\ Laura COSTA ${ }^{\dagger}$ \\ e-mail: enrique@sunal1.mat.ucm.es \\ e-mail: costa@cerber.mat.ub.es \\ DRAFT:5/4/98
}

\section{Introduction}

A well-known result of Horrocks (see [Ho]) states that a vector bundle on a projective space has not intermediate cohomology if and only if it decomposes as a direct sum of line bundles. There are two possible generalizations of this result to arbitrary varieties. The first one consists of giving a cohomological characterization of direct sums of line bundles. This has been done for quadrics and Grassmannians by Ottaviani (see O1, [02]) and for rank-two vector bundles on smooth hypersurfaces in $\mathbb{P}^{4}$ by Madonna (see Ma ).

The second generalization, to which we make some contribution in this paper, is to characterize vector bundles without intermediate cohomology. Besides the result of Horrocks for projective spaces, there is such a characterization for smooth quadrics due to Knörrer (see $[\mathrm{Kn}$ ). In fact, it was shown by Buchweitz, Greuel and Schreyer that only in the above two kind of varieties (projective spaces and quadrics) there is,

\footnotetext{
*Partially supported by DGICYT PB96-0659.

†Partially supported by DGICYT PB94-0850 and DGICYT PB96-0166.
} 
up to a twist by a line bundle, a finite number of indecomposable vector bundles without intermediate cohomology (see [BGS]). As a consequence, one should not expect to find a precise characterization of vector bundles without intermediate cohomology on arbitrary varieties. As far as we know, there is only a result in that direction, for the Grassmann variety $G(1,4)$ of lines in $\mathbb{P}^{4}$, due to Graña and the first author (see [AG]).

The present paper deals with vector bundles without intermediate cohomology on some Fano threefolds with the Betti number $b_{2}=1$. Specifically, we will work over a smooth cubic in $\mathbb{P}^{4}$, a smooth complete intersection of type $(2,2)$ in $\mathbb{P}^{5}$ and over a smooth intersection of the Grassmannian $G(1,4) \subset \mathbb{P}^{9}$ with three hyperplanes. For rank-two vector bundles, we give a complete classification (see Theorem 3.4). For arbitrary rank, we characterize which are the Chern classes of vector bundles without intermediate cohomology and verifying some general conditions (see Theorem 4.9).

Next we outline the structure of the paper. In section 2, we recall some general background. In section 3, we introduce some standard rank-two vector bundles on our Fano threefolds without intermediate cohomology, and we prove that any indecomposable rank-two vector bundle is, up to a twist, one of these. In section 4, we give the characterization of the Chern classes of "sufficiently general" vector bundles without intermediate cohomology and having arbitrary rank.

Acknowledgements: The authors want to express their gratitude to the Dipartimento di Matematica of the Università degli Studi di Milano, where most of the work has been developed. They also want to stress the important help that the Maple package Schubert, created by S. Katz and S.A. Strømme, has provided for some of the computations needed in this work.

\section{Generalities.}

For $d=3,4,5$, let $V_{d}$ be a Fano 3 -fold of degree $d$, index 2, with the Betti number $b_{2}=1$ and such that one of the two generators of its Picard group is spanned. Then, it has to be

$$
\begin{aligned}
& V_{3}=\text { a smooth cubic in } \mathbb{P}^{4} \\
& V_{4}=\text { a smooth complete intersection of type }(2,2) \text { in } \mathbb{P}^{5}
\end{aligned}
$$


$V_{5}=$ a smooth intersection of the Grassmannian $G(1,4) \subset \mathbb{P}^{9}$ with three hyperplanes.

Indeed, it is known (see e.g. [IS]) that there exist, up to a deformation type, only four Fano threefolds of index two, the above ones and a double covering $V_{2}$ of $\mathbb{P}^{3}$ ramified along a quartic surface.

Notation 2.1 Let $O_{V_{d}}(1)$ be the ample generator of $\operatorname{Pic}\left(V_{d}\right)$. In our situation, $O_{V_{d}}(1)$ is in fact very ample, and corresponds to the hyperplane section $H$ of $V_{d}$ in $\mathbb{P}^{d-1}$. Also, the canonical divisor of $V_{d}$ is given by $K=-2 H$. By a line on $V_{d}$ we mean a rational curve $C$ such that $C \cdot O_{V_{d}}(1)=1$ and we shall denote it by $L$, whereas a conic is a rational curve $C$ with $C \cdot O_{V_{d}}(1)=2$ and we shall denote it by $C$.

Since $H^{2}\left(V_{d}, \mathbb{Z}\right)$ is generated by the class of $H, H^{4}\left(V_{d}, \mathbb{Z}\right)$ by a line $L$, and $H^{6}\left(V_{d}, \mathbb{Z}\right)$ by a point $P$, we may identify the Chern classes $c_{i} \in H^{2 i}\left(V_{d}, \mathbb{Z}\right)$ of vector bundles with integers, namely the coefficients of $H, L$, and $P$. With this notation, $H L=1$, $H^{2}=d L$ and $H^{3}=d$.

Given $F$ a coherent sheaf on $V_{d}$ we will write $F(t)$ instead of $F \otimes O_{V_{d}}(t H)$.

Definition 2.2 A vector bundle $F$ on $V_{d}$ has not intermediate cohomology if and only if $H^{i}\left(V_{d}, F(t)\right)=0$, for all $t \in \mathbb{Z}$ and $i=1,2$.

We will end this section by recalling the formulas for Chern classes of a twist of a rank- $r$ vector bundle, the Riemann-Roch formula for rank- $r$ vector bundles on $V_{d}$ and the well-known regularity criterion of Castelnuovo-Mumford.

2.3 Given $F$ a rank- $r$ vector bundle on $V_{d}$ with Chern classes $c_{1}(F)=c_{1}, c_{2}(F)=c_{2}$ and $c_{3}(F)=c_{3}$ it holds:

$$
\begin{gathered}
c_{1}(F(t))=c_{1}+r t \\
c_{2}(F(t))=c_{2}+(r-1) t c_{1} d+\left(\begin{array}{l}
r \\
2
\end{array}\right) t^{2} d ; \\
c_{3}(F(t))=c_{3}+(r-2) c_{2} t+\left(\begin{array}{c}
r-1 \\
2
\end{array}\right) c_{1} t^{2} d+\left(\begin{array}{l}
r \\
3
\end{array}\right) t^{3} d ; \\
\chi(F)=\frac{d c_{1}^{3}-3 c_{1} c_{2}}{6}+\frac{d c_{1}^{2}-2 c_{2}}{2}+\frac{(d+3) c_{1}}{3}+\frac{c_{3}}{2}+r .
\end{gathered}
$$


In particular,

$$
\chi\left(O_{V_{d}}(t)\right)=\frac{d t^{3}}{6}+\frac{d t^{2}}{2}+\frac{(d+3) t}{3}+1 .
$$

2.4 Let $O(1)$ be an ample invertible sheaf on a variety $X$ which is generated by global sections. Let $F$ be a vector bundle with

$$
H^{i}(X, F(-i))=0, \quad i=1,2, \cdots, \operatorname{dim} X .
$$

Then,

i) $H^{i}(X, F(k))=0$, for any $i=1,2, \cdots, \operatorname{dim} X$ and $k \geq-i$;

ii) $F$ is generated by global sections.

\section{Rank-two vector bundles without intermediate cohomology}

The aim of this section is to characterize, up to twist by line bundles, rank-two vector bundles on $V_{d}$ without intermediate cohomology.

In the next examples we will summarize several facts about vector bundles on $V_{d}$ that will be used in the sequel. Some of these results are due to M. Szurek and J.A. Wiśniewski ([SW]).

Example 3.1 Let $L$ be a line in $V_{d}$. Serre's correspondence provides a vector bundle $S_{L}$ fitting in an exact sequence:

$$
0 \rightarrow O_{V_{d}} \rightarrow S_{L} \rightarrow I_{L} \rightarrow 0
$$

The rank-two vector bundle $S_{L}$ has Chern classes $\left(c_{1}, c_{2}\right)=(0,1)$, is semistable and has not intermediate cohomology. It holds that $S_{L}$ has only one section, whereas $S_{L}(1)$ is generated by global sections. The latter comes from the exact sequence (11), the fact that $O_{V_{d}}(1)$ and $I_{L}(1)$ are generated by its sections and the vanishing of $H^{1}\left(O_{V_{d}}(1)\right)$. 
Example 3.2 If $C$ is a conic in $V_{d}$, let $S_{C}$ be the rank-two vector bundle obtained from Serre's construction and fitting in a non-trivial extension:

$$
0 \rightarrow O_{V_{d}}(-1) \rightarrow S_{C} \rightarrow I_{C} \rightarrow 0
$$

It holds that $S_{C}$ is stable and generated by its global sections. It has not intermediate cohomology and has Chern classes $\left(c_{1}, c_{2}\right)=(-1,2)$.

When $d=4, S_{C}$ is related to the spinor bundles of the quadrics containing $V_{4}$. More precisely, let $\Pi$ be the unique plane containing $C$. Then, only one quadric $Q_{4}$ containing $V_{4}$ contains $\Pi$. If $Q_{4}$ is smooth, then $S_{C}(1)$ is the restriction to $V_{d}$ of one of the spinor bundles of $Q_{4}$. In other words, if we identify $Q_{4}$ with the Grassmann variety $G(1,3)$ of lines in $\mathbb{P}^{3}$, then $S_{C}(1)$ is the restriction to $V_{4}$ of one of the universal bundles of $G(1,3)$. On the other hand, when $d=5, S_{C}(1)$ is the restriction to $V_{5}$ of the rank-two universal quotient bundle on $G(1,4)$.

Example 3.3 Using again Serre's correspondence, an elliptic curve $E$ of degree $d+2$ gives rise to a stable, rank-two vector bundle $S_{E}$, with Chern classes $\left(c_{1}, c_{2}\right)=(0,2)$, without intermediate cohomology and appearing in an exact sequence:

$$
0 \rightarrow O_{V_{d}}(-1) \rightarrow S_{E} \rightarrow I_{E}(1) \rightarrow 0
$$

It also holds that $S_{E}(1)$ is generated by its global sections.

Next we will prove that the above examples are essentially the only rank-two vector bundles on $V_{d}$ without intermediate cohomology.

Theorem 3.4 An indecomposable rank-two vector bundle $F$ on $V_{d}$ with $d=3,4,5$ has not intermediate cohomology if and only if it is a twist of either $S_{L}$, or $S_{C}$, or $S_{E}$.

Proof: Let $F$ be an indecomposable rank-two vector bundle on $V_{d}$ with Chern classes $c_{1}(F)=c_{1}$ and $c_{2}(F)=c_{2}$, which has not intermediate cohomology. Without loss of generality we can assume that $F(-1)$ has no sections while $F$ has. Hence, any section of $F$ gives rise to an exact sequence:

$$
0 \rightarrow O_{V_{d}} \rightarrow F \rightarrow I_{D}\left(c_{1}\right) \rightarrow 0
$$

where $D$ is a scheme of pure dimension one. Observe that it cannot be $D=\emptyset$, since $\operatorname{Ext}^{1}\left(O_{V_{d}}\left(c_{1}\right), O_{V_{d}}\right) \cong H^{1}\left(O_{V_{d}}\left(-c_{1}\right)\right)=0$, and this would imply that $F$ decomposes.

Claim: 


$$
F\left(2-c_{1}\right) \text { is generated by its global sections and therefore } c_{1} \leq 2 .
$$

Proof of the Claim: Using Serre's duality and the natural identification $F^{*} \cong F\left(-c_{1}\right)$ we have:

$$
h^{3}\left(F\left(-1-c_{1}\right)\right)=h^{0}(F(-1))=0 .
$$

Hence, since $F$ has not intermediate cohomology, using Castelnuovo-Mumford criterion 2.4, we obtain the claim.

We will prove the theorem distinguing two cases.

Case A: Assume that $c_{1} \leq 0$. From Serre's duality and (đ) twisted with $O_{V_{d}}\left(-c_{1}-\right.$ $1)$, we get that the only cohomology of $F(-1)$ is $h^{3}(F(-1))=h^{0}\left(F\left(-c_{1}-1\right)\right)=$ $h^{0}\left(O_{V_{d}}\left(-c_{1}-1\right)\right)$. Computing $\chi(F(-1))$ and $\chi\left(O_{V_{d}}\left(-c_{1}-1\right)\right)$ from 2.3 we get:

$$
\begin{gathered}
\chi(F(-1))=\frac{c_{1}^{3} d}{6}-\frac{c_{1} c_{2}}{2}-\frac{d c_{1}}{6}+c_{1} ; \\
\chi\left(O_{V_{d}}\left(-c_{1}-1\right)\right)=-\frac{c_{1}^{3} d}{6}+\frac{d c_{1}}{6}-c_{1} .
\end{gathered}
$$

Hence, from $\chi\left(O_{V_{d}}\left(-c_{1}-1\right)\right)=-\chi(F(-1))$ we obtain the identity $c_{1} c_{2}=0$. Since $D \neq \emptyset$, we have $c_{2} \neq 0$, and hence $c_{1}=0$. Again from 2.3 and the fact that $F$ has not intermediate cohomology we get $2-c_{2}=\chi(F)=h^{0}(F) \geq 1$, from which $c_{2}=1$. This means that $D$ is a line $L$, and therefore $F \cong S_{L}$.

Case $B$ : If $c_{1}>0$, we know from the claim that the only possibilities are $c_{1}=1,2$. In fact, this can be obtained, together with a much more precise information, with the same techniques as in case A. Indeed, we have now that $h^{0}\left(F\left(-c_{1}\right)\right)=0$, so that $h^{3}(F(-1))=h^{3}(F(-2))=0$. Therefore, $\chi(F(-1))=\chi(F(-2))=0$. Again, using 2.3, we have

$$
\chi(F(-2))=\frac{c_{1}^{3} d}{6}-\frac{c_{1} c_{2}}{2}+c_{1}-\frac{d c_{1}^{2}}{2}+c_{2}-2+\frac{d c_{1}}{3} .
$$

Comparing with the above expression for $\chi(F(-1))$, we obtain that $c_{2}-\frac{d c_{1}^{2}}{2}-2+\frac{d c_{1}}{2}=$ 0 , and therefore the only possibilities are $\left(c_{1}, c_{2}\right)=(1,2),(2, d+2)$.

In the first case, $D$ has degree $c_{2}=2$, and by Serre's correspondence $\omega_{D} \cong$ $O_{D}\left(c_{1}-2\right)=O_{D}(-1)$, which means that $D$ is a plane conic $C$ (maybe singular or even non-reduced). Therefore, $F \cong S_{C}(1)$. 
In the second case, by the claim, $F$ is then generated by its global sections. This implies that a general section of $F$ vanishes on a smooth curve $D$. Since $\omega_{D}=$ $O_{D}\left(c_{1}-2\right)=O_{D}$, this means that $D$ is an elliptic curve. Its degree is $c_{2}=d+2$. Therefore, $F \cong S_{E}(1)$ where $S_{E}$ is the vector bundle on $V_{d}$ associated to an elliptic curve $E$ of degree $d+2$.

\section{Vector bundles of higher rank without interme- diate cohomology}

In this section we will study vector bundles of rank $r \geq 3$ without intermediate cohomology. While in the previous section we gave a complete classification, in this

section we will just characterize the possible Chern classes. We will also need to make some general assumptions.

Convention: For simplicity, we will frequently use $(\star)$ to denote the following conditions on a rank- $r$ vector bundle $F$ on $V_{d}$ :

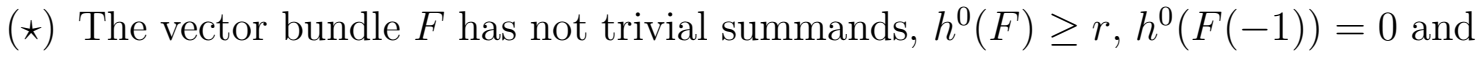
$F$ has $r-1$ sections whose dependency locus has codimension two.

Lemma 4.1 Let $F^{\prime}$ and $F^{\prime \prime}$ be two vector bundles on $V_{d}$ with respective ranks $r^{\prime}$ and $r^{\prime \prime}$. If $F^{\prime}$ and $F^{\prime \prime}$ verify $(\star)$, then also $F^{\prime} \oplus F^{\prime \prime}$ verifies $(\star)$.

Proof: It is clear from the definition that, since $F^{\prime}$ and $F^{\prime \prime}$ verify $(\star)$, then their global sections generate them up to probably a subvariety of codimension at least two. Therefore, the same holds for $F^{\prime} \oplus F^{\prime \prime}$. This implies that the dependency locus of $r^{\prime}+r^{\prime \prime}-1$ general sections of $F^{\prime} \oplus F^{\prime \prime}$ has codimension two. From this, it follows immediately that $F^{\prime} \oplus F^{\prime \prime}$ verifies $(\star)$.

Before stating and proving our main theorem, we will give a series of examples. Eventually this list will become the complete list for small rank.

Example 4.2 Let $D$ be a rational curve of degree $r \leq d+1$ in $V_{d}$. Serre's construction yields a rank- $r$ vector bundle $F$ fitting in an exact sequence

$$
0 \rightarrow O_{V_{d}}^{r-1} \rightarrow F \rightarrow I_{D}(1) \rightarrow 0
$$


Since $D$ is projectively normal in $\mathbb{P}^{d+1}$ if and only if $r \leq d+1$, then $F$ has not intermediate cohomology. From the above exact sequence it holds that $c_{1}(F)=1$, $c_{2}(F)=r$ and $c_{3}(F)=r-2$, and also $h^{0}(F)=d$. Notice that, when $d=5$ and $r=3, F$ is the restriction to $V_{5}$ of the dual of the rank-three universal subbundle on $G(1,4)$, and in particular it is generated by its global sections.

Example 4.3 Take $F$ to be the rank-three vector bundle given by the above example when $r=3$. Then $\wedge^{2} F \cong F^{*}(1)$ has not intermediate cohomology, has general sections and Chern classes $c_{1}=2, c_{2}=d+3$ and $c_{3}=2$. Since, for $d=5, F$ is generated by its global sections, then the same holds for $\bigwedge^{2} F$.

Example 4.4 Consider the elliptic curve $E$ given in Example 3.3. Exact sequence (3) implies that there is a section $s \in H^{0}\left(S_{L}(2)\right)$ vanishing on $E$ and not vanishing on a divisor of $V_{d}$. If $D^{\prime}$ is the residual curve of $E$ inside the zero locus of $s$, it turns out that $D^{\prime}$ has degree $3 d-1$ and (arithmetic) genus $2 d-2$. The standard mapping cone construction (see for instance Lemma 3.2 in AS) provides an exact sequence

$$
0 \rightarrow S_{E}(-3) \rightarrow O_{V_{d}}(-2) \oplus S_{L}(-2) \rightarrow I_{D^{\prime}} \rightarrow 0
$$

We use now this exact sequence to see that there is a section $s^{\prime} \in H^{0}\left(S_{C}(3)\right)$ vanishing on $D^{\prime}$ and not vanishing on a divisor of $V_{d}$. We have now the followig exact sequences for $D$, the residual curve of $D^{\prime}$ inside the zero locus of $s^{\prime}$ :

$$
0 \rightarrow S_{L}(-3) \oplus O_{V_{d}}(-3) \rightarrow S_{E}(-2) \oplus S_{C}(-2) \rightarrow I_{D} \rightarrow 0
$$

and its dual

$$
0 \rightarrow O_{V_{d}} \rightarrow S_{E}(-2) \oplus S_{C}(3) \rightarrow S_{L}(3) \oplus O_{V_{d}}(3) \rightarrow \omega_{D} \rightarrow 0
$$

The curve $D$ has degree $3 d+3$ and arithmetic genus $2 d+4$. From the exact sequence (6) tensored with $O_{V_{d}}(-3)$ it follows that $h^{0}\left(\omega_{D}(-1)\right)=2$, so that Serre's construction yields a rank-three vector bundle $F$ with Chern classes $c_{1}=3, c_{2}=3 d+3, c_{3}=d+3$ and fitting in an exact sequence

$$
0 \rightarrow O_{V_{d}}^{2} \rightarrow F \rightarrow I_{D}(3) \rightarrow 0
$$

Comparing with (5) it easily follows that $H^{0}(F(-1))=0, H^{1}(F(l))=0$ for any $l \in Z$, and $H^{2}(F(l))=0$ for any $l \geq-1$. It also follows from Serre's construction 
that $H^{2}(F(-2))=0$. Hence, Mumford-Castelnuovo criterion applied to $F^{*}(2)$ will imply that $F$ has not intermediate cohomology as soon as we prove that $h^{1}\left(F^{*}(1)\right)=$ $h^{2}(F(-3))=0$. We observe that, from the exact sequences (6) and the dual of (7) and our construction, there is a commutative diagram

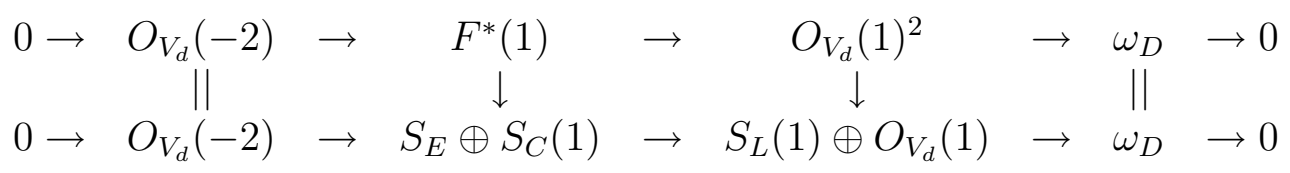

The vanishing we want to prove reduces to the surjectivity of the map $H^{0}\left(O_{V_{d}}(1)^{2}\right) \rightarrow$ $H^{0}\left(\omega_{D}\right)$, but this is proved by just taking cohomology in the above diagram.

Notice that the above construction does not imply a priori that the curve $D$ is smooth. However, once the vector bundle $F$ is constructed, it follows by the Castelnuovo-Mumford criterion that $F$ is generated by its global sections. Hence, the dependency locus of two general sections of $F$ is a smooth curve $D$.

When $d=5$, such a vector bundle $F$ can be constructed in a more direct way. Indeed, in this case $S_{C}(1)$ is the restriction to $V_{5}$ of the universal rank-two quotient bundle $Q$ on $G(1,4)$. Since $S^{2} Q$ has not intermediate cohomology, neither $F=$ $S^{2}\left(S_{C}(1)\right)$ has. It follows at once that $F$ is a rank-three vector bundle, generated by its global sections and its Chern classes are $c_{1}=3, c_{2}=18$ and $c_{3}=8$.

Example 4.5 Let $G$ be the rank-three vector bundle obtained in Example 4.3 when $d=5$. Since $G$ was generated by its global sections and $h^{0}(G)=10$, then there is an exact sequence

$$
0 \rightarrow K \rightarrow O_{V_{5}}^{10} \rightarrow G \rightarrow 0
$$

where $K$ is defined as a kernel. It follows that $F=K^{*}$ is a rank-seven vector bundle without intermediate cohomology and generated by its global sections. It also holds that $c_{1}(F)=2, c_{2}(F)=12$ and $c_{3}(F)=10$. 
Table 4.6 From Lemma 4.1 and the above examples, we can give the following list of rank- $r$ vector bundles $F_{r, c}$ without intermediate cohomology, verifying $(\star)$ and with $c_{1}\left(F_{r, c}\right)=c$

\begin{tabular}{|c|c|c|c|}
\hline$d$ & $r$ & $\left(c, c_{2}, c_{3}\right)$ & $F_{r, c}$ \\
\hline \hline $3,4,5$ & 3 & $(1,3,1)$ & Example 4.2 \\
\hline $3,4,5$ & 3 & $(2, d+3,2)$ & Example 4.3 \\
\hline $3,4,5$ & 3 & $(3,3 d+3, d+3)$ & Example 4.4 \\
\hline 4,5 & 4 & $(1,4,2)$ & Example $\overline{4.2}$ \\
\hline $3,4,5$ & 4 & $(2, d+4,4)$ & $S_{C}(1) \oplus S_{C}(1)$ \\
\hline $3,4,5$ & 4 & $(3,3 d+4, d+6)$ & $S_{C}(1) \oplus S_{E}(1)$ \\
\hline $3,4,5$ & 4 & $(4,6 d+4,4 d+8)$ & $S_{E}(1) \oplus S_{E}(1)$ \\
\hline 5 & 5 & $(1,5,3)$ & Example 4.2 \\
\hline $3,4,5$ & 5 & $(2, d+5,6)$ & $S_{C}(1) \oplus F_{3,1}$ \\
\hline $3,4,5$ & 5 & $(3,3 d+5, d+9)$ & $S_{C}(1) \oplus F_{3,2}$ \\
\hline $3,4,5$ & 5 & $(4,6 d+5,4 d+2)$ & $S_{C}(1) \oplus F_{3,3}$ \\
\hline $3,4,5$ & 5 & $(5,10 d+5,10 d+15)$ & $S_{E}(1) \oplus F_{3,3}$ \\
\hline $3,4,5$ & 6 & $(2, d+6,8)$ & $F_{3,1} \oplus F_{3,1}$ \\
\hline $3,4,5$ & 6 & $(3,3 d+6, d+12)$ & $S_{C}(1) \oplus S_{C}(1) \oplus S_{C}(1)$ \\
\hline $3,4,5$ & 6 & $(4,6 d+6,4 d+16)$ & $S_{C}(1) \oplus S_{C}(1) \oplus S_{E}(1)$ \\
\hline $3,4,5$ & 6 & $(5,10 d+6,10 d+20)$ & $S_{C}(1) \oplus S_{E}(1) \oplus S_{E}(1)$ \\
\hline $3,4,5$ & 6 & $(6,15 d+6,20 d+24)$ & $S_{E}(1) \oplus S_{E}(1) \oplus S_{E}(1)$ \\
\hline 4,5 & 7 & $(2, d+7,10)$ & $F_{4,1} \oplus F_{3,1}$ \\
\hline $3,4,5$ & 7 & $(3,3 d+7, d+15)$ & $S_{C}(1) \oplus S_{C}(1) \oplus F_{3,1}$ \\
\hline $3,4,5$ & 7 & $(4,6 d+7,4 d+20)$ & $S_{C}(1) \oplus S_{E}(1) \oplus F_{3,1}$ \\
\hline $3,4,5$ & 7 & $(5,10 d+7,10 d+25)$ & $S_{E}(1) \oplus S_{E}(1) \oplus F_{3,1}$ \\
\hline $3,4,5$ & 7 & $(6,15 d+7,20 d+30)$ & $S_{E}(1) \oplus S_{E}(1) \oplus F_{3,2}$ \\
\hline $3,4,5$ & 7 & $(7,21 d+7,35 d+35)$ & $S_{E}(1) \oplus S_{E}(1) \oplus F_{3,3}$ \\
\hline
\end{tabular}

Remark 4.7 Notice that in the above table, vector bundles with the same invariants can be constructed in different ways. Moreover, in some cases it is possible to consider vector bundles given by non-trivial extensions instead of direct sums. For instance, 
it is immediate to see that $\operatorname{Ext}^{1}\left(S_{E}(1), S_{E}(1)\right) \neq 0$. In fact, the dimension of this space of extensions is five, the same as the dimension of the space of elliptic curves of degree $d+2$ contained in $V_{d}$. Clearly, a vector bundle obtained from a non-trivial extension also verifies condition $(\star)$ and has not intermediate cohomology.

Remark 4.8 In case of rank $r=3$, it is clear that the vector bundles in the above list are indecomposable, since otherwise any direct summand of rank one would be necessarely trivial. In case $r=4$ and $c_{1}=1$ (even when $d=3$, as constructed in Example 4.2) the corresponding vector bundle in the above list is also indecomposable. Indeed, we can rule out (using the classification given in the previous section) all its possible rank-two direct summands. What seems to be difficult is to decide in general when it is possible to find an indecomposable vector bundle without intermediate cohomology and having some of the above invariants. This is so even when we know that we can take non-trivial extensions as in the above remark.

We can now state and prove the main result of this section.

Theorem 4.9 Let $r \geq 3$ be an integer. There exists a rank-r vector bundle $F$ on $V_{d}$ without intermediate cohomology, verifying $(\star)$ and with Chern classes $c_{1}(F)=c_{1}$, $c_{2}(F)=c_{2}$ and $c_{3}(F)=c_{3}$ if and only if

$$
\begin{gathered}
\text { (i) } c_{2}=\frac{d c_{1}^{2}}{2}+r-\frac{d c_{1}}{2} ; \\
\text { (ii) } c_{3}=-2 c_{1}+c_{1} r-\frac{d c_{1}^{2}}{2}+\frac{d c_{1}^{3}}{6}+\frac{d c_{1}}{3} ; \\
\text { (iii) } \frac{r}{d} \leq c_{1} \leq r .
\end{gathered}
$$

Moreover, in this situation, the dependency locus of $r-1$ general sections of such $F$ is a curve $D$ of degree $c_{2}$ and arithmetic genus

$$
p_{a}(D)=\left(c_{1}-1\right)(r-1)-d c_{1}^{2}+\frac{d c_{1}^{3}}{3}+\frac{2 d c_{1}}{3} .
$$


Proof: Let $F$ be a rank- $r$ vector bundle on $V_{d}$ verifying $(\star)$, with Chern classes $c_{1}(F)=c_{1}, c_{2}(F)=c_{2}$ and $c_{3}(F)=c_{3}$, which has not intermediate cohomology. By assumption, $F$ has $r-1$ sections whose dependency locus has codimension two. These sections of $F$ give rise to an exact sequence:

$$
0 \rightarrow O_{V_{d}}^{r-1} \rightarrow F \rightarrow I_{D}\left(c_{1}\right) \rightarrow 0
$$

where $D$ is a scheme of pure dimension one. Observe that it cannot be $D=\emptyset$, since $\operatorname{Ext}^{1}\left(O_{V_{d}}\left(c_{1}\right), O_{V_{d}}{ }^{r-1}\right) \cong H^{1}\left(O_{V_{d}}\left(-c_{1}\right)\right)^{\oplus(r-1)}=0$, and this would imply that (8) splits, and hence $F$ would have a trivial summand.

Let us see that $c_{1}>0$. Indeed, assume that $c_{1} \leq 0$. From Serre's duality and (8) twisted by $O_{V_{d}}(-1)$, the only cohomology of $F(-1)$ is $h^{3}(F(-1))=h^{3}\left(O_{V_{d}}\left(c_{1}-1\right)\right)$. Since $c_{1}(F(-1))=c_{1}-r, c_{2}(F(-1))=c_{2}-(r-1) c_{1} d+\left(\begin{array}{c}r \\ 2\end{array}\right) d$ and $c_{3}(F(-1))=$ $c_{3}-(r-2) c_{2}+\left(\begin{array}{c}r-1 \\ 2\end{array}\right) c_{1} d-\left(\begin{array}{c}r \\ 3\end{array}\right) d$, computing $\chi(F(-1))$ and $\chi\left(O_{V_{d}}\left(c_{1}-1\right)\right)$ from 2.3 we get:

$$
\begin{gathered}
\chi(F(-1))=\frac{c_{1}^{3} d}{6}-\frac{c_{1} c_{2}}{2}-\frac{d c_{1}}{6}+c_{1}+\frac{c_{3}}{2} ; \\
\chi\left(O_{V_{d}}\left(c_{1}-1\right)\right)=\frac{c_{1}^{3} d}{6}-\frac{d c_{1}}{6}+c_{1} .
\end{gathered}
$$

Hence, from $\chi\left(O_{V_{d}}\left(c_{1}-1\right)\right)=\chi(F(-1))$ we obtain the identity $c_{3}-c_{1} c_{2}=0$.

Similarly, we have $\chi\left(F\left(-c_{1}\right)\right)=(r-1) \chi\left(O_{V_{d}}\left(-c_{1}\right)\right)$, which is equivalent to:

$$
\begin{gathered}
\left(-\frac{(r-1) c_{1}^{3}}{6}-\frac{(r-1) c_{1}}{3}+\frac{(r-1) c_{1}^{2}}{2}\right) d+\frac{c_{1} c_{2}}{2}-c_{2}+\frac{c_{3}}{2}-r c_{1}+c_{1}+r= \\
(r-1)\left(\left(-\frac{c_{1}^{3}}{6}+\frac{c_{1}^{2}}{2}-\frac{c_{1}}{3}\right) d-c_{1}+1\right)
\end{gathered}
$$

and $\chi\left(F\left(-c_{1}-1\right)\right)=(r-1) \chi\left(O_{V_{d}}\left(-c_{1}-1\right)\right.$, which is equivalent to:

$$
\begin{gathered}
\left(-\frac{(r-1) c_{1}^{3}}{6}+\frac{(r-1) c_{1}}{6}\right) d+\frac{c_{1} c_{2}}{2}+\frac{c_{3}}{2}-r c_{1}+c_{1}= \\
(r-1)\left(\left(-\frac{c_{1}^{3}}{6}+\frac{c_{1}}{6}\right) d-c_{1}\right)
\end{gathered}
$$

from which we respectively obtain the identities $c_{1} c_{2}+c_{3}=c_{2}-1$ and $c_{1} c_{2}+c_{3}=0$. Putting together the three identities, we get $c_{1}=c_{3}=0$ and $c_{2}=1$, so that in particular $D$ is a line $L$. 
The dual of (8) is

$$
0 \rightarrow O_{V_{d}}\left(-c_{1}\right) \rightarrow F^{*} \rightarrow O_{V_{d}}^{\oplus(r-1)} \rightarrow \omega_{D}\left(2-c_{1}\right) \rightarrow 0 .
$$

Since $\omega_{D}\left(2-c_{1}\right)$ is trivial in our case, it follows that $F^{*}$ has $r-2$ independent sections such that the composed map $O_{V_{d}}{ }^{\oplus(r-2)} \rightarrow F^{*} \rightarrow O_{V_{d}}{ }^{(r-1)}$ defines a direct summand. But this implies that $F \cong O_{V_{d}} \oplus(r-2) \oplus S_{L}$, which is a contradiction as $r \geq 3$. Hence, $c_{1}>0$.

Since $c_{1}>0$, we have that $h^{3}(F(-1))=h^{3}(F(-2))=0$ (the latter equality is equivalent to $h^{0}\left(F^{*}\right)=0$, which is obtained from (9) as above, by using that $F$ has no trivial summands). Therefore, $\chi(F(-1))=\chi(F(-2))=0$. Again, using 2.3, we have:

$$
\begin{gathered}
\chi(F(-1))=\frac{c_{1}^{3} d}{6}-\frac{c_{1} c_{2}}{2}-\frac{d c_{1}}{6}+c_{1}+\frac{c_{3}}{2} \\
\chi(F(-2))=\frac{c_{1}^{3} d}{6}-\frac{c_{1} c_{2}}{2}+c_{1}-\frac{d c_{1}^{2}}{2}+c_{2}-r+\frac{d c_{1}}{3}+\frac{c_{3}}{2} .
\end{gathered}
$$

The vanishing of the above two terms immediately implies that $c_{2}(F)$ and $c_{3}(F)$ verify the identities $(i)$ and $(i i)$.

Using once more 2.3, we obtain that $-h^{3}(F(-3))=\chi(F(-3))=d\left(c_{1}-r\right)$. This implies that $c_{1} \leq r$. The other inequality in (iii) follows from the fact that $h^{0}(F) \geq$ $r-1$, Riemann-Roch equality 2.3 and the identities $(i)$ and $(i i)$.

Finally, the genus of the curve $D$ is $\chi\left(I_{D}\right)$, which can be computed from (8), and it is

$$
\left(c_{1}-1\right)(r-1)-d c_{1}^{2}+\frac{d c_{1}^{3}}{3}+\frac{2 d c_{1}}{3} .
$$

Conversely, let $r, c_{1}, c_{2}$ and $c_{3}$ be integers verifying $(i),(i i)$ and (iii). We will prove by induction on $r$ that there exists a rank- $r$ vector bundle $F$ on $V_{d}$ without intermediate cohomology, verifying $(\star)$ and with Chern classes $c_{1}(F)=c_{1}, c_{2}(F)=c_{2}$ and $c_{3}(F)=c_{3}$. For $r \leq 7$ this is showed in Table 4.6, so let us assume $r \geq 8$. In this case, the proof goes as for $r \leq 7$, using Lemma 4.1. We will distinguish three cases:

Case $A$ ) If $c_{1}=r$, we can take $F=S_{E}(1) \oplus F^{\prime}$, where $F^{\prime}$ is a rank- $(r-2)$ vector bundle on $V_{d}$ without intermediate cohomology, verifying $(\star)$ and with first Chern class $c_{1}\left(F^{\prime}\right)=r-2$. Indeed, such $F^{\prime}$ can be constructed by induction hypothesis. 
Case $B$ ) If $\frac{r-2}{d}+1 \leq c_{1} \leq r-1$, we take $F=S_{C}(1) \oplus F^{\prime}$, where $F^{\prime}$ is a rank- $(r-2)$ vector bundle on $V_{d}$ without intermediate cohomology, verifying $(\star)$ and with first Chern class $c_{1}\left(F^{\prime}\right)=c_{1}-1$. This vector bundle $F^{\prime}$ exists by induction hypothesis, since $F^{\prime}$ verifies $(i i i)$.

Case $C$ ) If $\frac{r}{d} \leq c_{1} \leq \frac{r-1}{d}+1$, we take now $F=F^{\prime} \oplus F^{\prime \prime}$, where $F^{\prime}$ and $F^{\prime \prime}$ are vector bundles on $V_{d}$ of respective ranks $r-d$ and $d$ without intermediate cohomology, verifying $(\star)$ and with Chern classes $c_{1}\left(F^{\prime}\right)=c_{1}-1, c_{1}\left(F^{\prime \prime}\right)=1$. Once again, $F^{\prime}$ verifies the inductive assumption since $r \geq 8$, and $F^{\prime \prime}$ was constructed in Example 4.2.

Remark 4.10 If we do not make the assumption that our vector bundles have at least $r$ independent sections, the same proof is clearly valid to see that in that case $(i)$ and (ii) holds, and instead of (iii) we would have that $\frac{r-1}{d} \leq c_{1} \leq r$. What presents some difficulty is to prove the actual existence of vector bundles with those invariants. This is due to the fact that we cannot make use of Lemma 4.1. In fact, we are still able to prove the existence of most of them, but some cases still remain unknown to us.

Remark 4.11 Our results are in fact valid over any Fano threefold of degree $d$ and index two and arbitrary Betti number. In that case, the only assumption we need on the vector bundles is their determinant to be a multiple of $O_{V_{d}}(1)$, the hyperplane section of $V_{d}$ in $\mathbb{P}^{d-1}$. 


\section{References}

[AG] E. Arrondo; B. Graña; Vector bundles on $G(1,4)$ without intermediate cohomology; Preprint-1998.

[AS] E. Arrondo; I. Sols; On congruences of lines in the projective space; Mém. Soc. Math. France 50 (1992).

[BGS] R.O. Buchweitz; G.M. Greuel; F.O. Schreyer; Cohen-Macaulay modules on hypersurface singularities II, Invent. Math. 88 (1987), 165-182.

[Ho] G. Horrocks; Vector bundles on the punctured spectrum of a ring, Proc. London Math. Soc. (3) 14 (1964), 689-713.

[Is] V.A. Iskovskih; Fano 3-Folds, I, Math. USSR Izvestija 11 (1977), 485-527.

[Kn] H. Knörrer;Cohen-Macaulay modules on hypersurface singularities I, Invent. Math. 88 (1987), 153-164.

[Ma] C. Madonna; A splitting criterion for rank 2 vector bundles on hypersurfaces in $P^{4}$, to appear in Rendiconti di Torino.

[O1] G. Ottaviani; Critères de scindage pour les fibrés vectoriels sur les grassmannianes et les quadriques, C.R. Acad. Sci. Paris, t. 305, Série I (1987), 257-260.

[O2] G. Ottaviani; Some extensions of Horrocks criterion to vector bundles on Grassmannians and quadrics, Annali Mat. Pura Appl. (IV) 155 (1989), 317-341.

[SW] M. Szurek; J.A. Wiśniewski; Conics, conic fibrations and stable vector bundles of rank 2 on some Fano threefolds, Rev. Roumaine Math. Pures Appl. 38 (1993), 729-741. 